\title{
Non-coronary sinus of Valsalva aneurysm diagnosed after a road traffic accident
}

\author{
Â Ribeiro, F Fernandes, A Costeira, A Simões, P Rodrigues
}

\begin{abstract}
A 38 year old man with a huge unruptured sinus of Valsalva aneurysm, complicated with severe valvar aortic regurgitation, is described. The aneurysm was detected by echocardiography in the asymptomatic patient who presented with an intense precordial diastolic rumble after a road traffic accident. The patient had successful surgery for the aneurysm and aortic valve replacement. Possible aetiologies for the aneurysm and a brief revision of clinical aspects and treatment are discussed. (Heart 1999;82:e5)
\end{abstract}

Keywords: sinus of Valsalva aneurysm; valvar disorders; trauma

A 38 year old man was admitted to hospital after a road traffic accident. He had multiple lesions including thoracic trauma with rib fracture and deceleration injuries - that is, plexus brachial stretching. Although he had no previous cardiac complications, a heart murmur was detected, and he was therefore referred to the cardiology department. Examination revealed divergent arterial pressure, hyperkinetic arterial pulse, and a long diastolic heart murmur. Transthoracic echocardiography with Doppler colour flow mapping showed normal cardiac chambers, dilatation of the aortic root (the sac-

Accepted for publication 27 April 1999

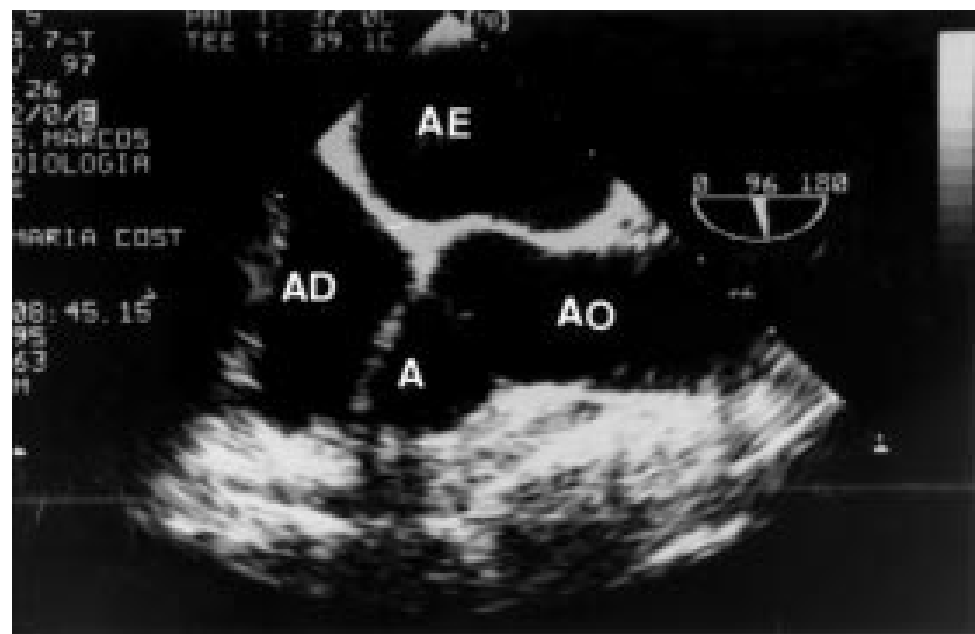

Figure 1 Multiplane transoesophageal echocardiogram showing a huge sinus of Valsalva aneurysm $(A)$ which appears to involve the right coronary and non-coronary sinuses. $A E$, left atrium; $A D$, right atrium; $A O$, aorta.

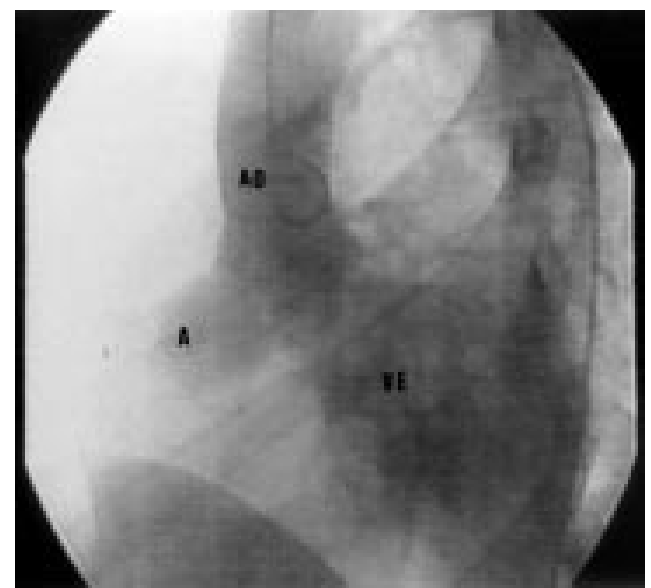

Figure 2 Aortogram showing the sinus of Valsalva aneurysm (A). AO, aorta; VE, left ventricle.

cular image suggested a sinus of Valsalva aneurysm), morphologically normal valves, good left ventricular systolic function, and a moderate to severe aortic regurgitation with no other abnormal flows. A transoesophageal echocardiogram was taken for a better image and showed a huge unruptured aneurysm (fig 1), which appeared to involve two Valsalva sinuses (non-coronary and right), and severe aortic regurgitation. Cardiac catheterisation indicated a similar pathology to that seen in the echocardiogram; other coronary vessels were normal (fig 2). Surgery revealed the aneurysm to be non-coronary and it was therefore corrected by direct sewing; the aortic valve was replaced with a St Jude prosthesis. There were no postoperative complications and the patient was discharged on the seventh day.

An echocardiogram taken after the operation shows the para-aortic image in the right coronary cusp, and corresponds to the sinus of Valsalva aneurysm without interior flow (fig 3). The aortic mechanical prosthesis had normal gradients and the cardiac chambers had normal dimensions; systolic left ventricular function was maintained and the patient was free from cardiac symptoms.

\section{Discussion}

Sinus of Valsalva aneurysms are rare among cardiac surgery patients and it is extremely unusual to find them unruptured or asymptomatic. They are saccular pathological dilata- 


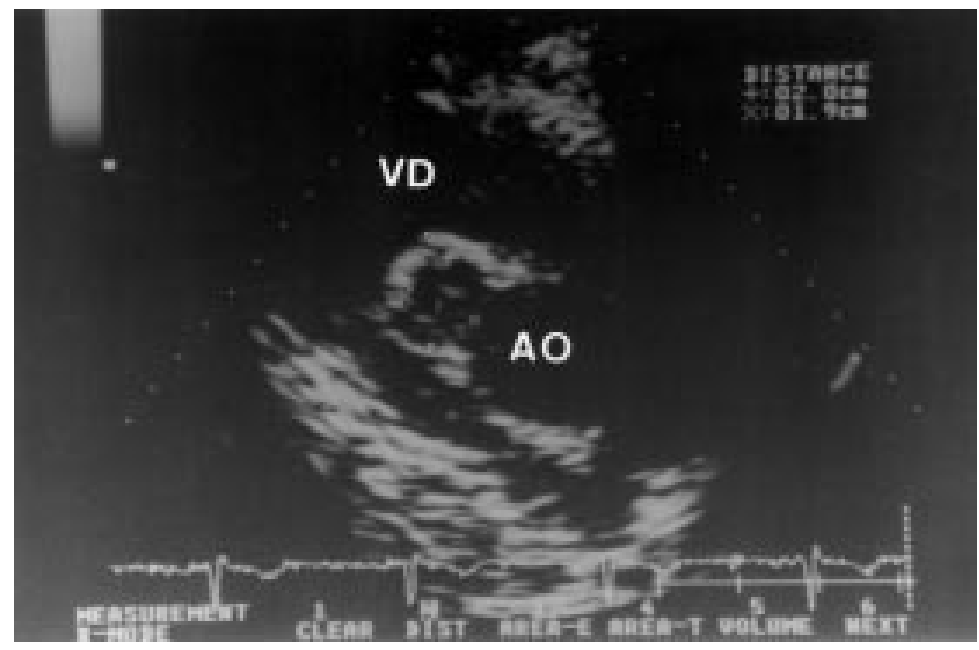

Figure 3 Postoperative transthoracic echocardiogram, parasternal short axis: para-aortic image is in the right coronary area; maximum dimensions $2 \mathrm{~cm} \times 1.9 \mathrm{~cm}$, corresponding to the obliterated sinus of Valsalva aneurysm. VD, right ventricle; $A O$, aorta.

tions of the aortic sinus outlined by a thin wall, caused by a lack of elastic or muscular tissue in the aortic root which causes the media of the aortic root and the fibrous annulus of the aortic valve to separate; they are usually congenital in origin. They are frequently found in the area of the right sinus of Valsalva, but are less frequent in the non-coronary sinuses and are rarely found at the left coronary cusp. They are also often associated with the other congenital cardiac malformations - for example, ventricular septal defects are present in $30-60 \%$ of cases, and aortic valve anomalies (bicuspid valve or prolapse) are seen in $10 \% .^{1-3}$

Aneurysms can be caused by bacterial endocarditis, trauma, degeneration of the aortic root, medionecrosis, and atherosclerosis, ${ }^{2-4}$ and can involve multiple sinuses of Valsalva (10\%) and the ascending aorta and more extensive portions of the aortic root. ${ }^{2}$ In most cases, sinus of Valsalva aneurysms are diagnosed by transthoracic echocardiography because it enables the aortic root to be seen clearly. Doppler colour flow echocardiography is the best technique for the detection of complications - for example, aneurysm rupture, but sometimes transoesophageal echocardiography is more appropriate as it provides a clearer image of a fistulised aneurysm. ${ }^{56}$

The most frequent complication is the rupture in the cardiac chambers (generally the right cavities) which usually occurs in men aged between 30 and 40 and develops into gradual or acute heart failure, with the presence of a harsh continuous murmur in the precordium. Fistulisation is rarely asymptomatic. Other complications of sinus of Valsalva aneurysms are aneurysm infection, obstruction of the right ventricle outflow tract, extension of the interventricular septum leading to malignant arrhythmias and conduction disturbances, an intramural thrombus causing obstruction of coronary ostia, and myocardial ischaemia. ${ }^{7}$ Ruptures in non-congenital cases are usually fatal, and occur in the pericardial or pleural cavity and, less frequently, in the right cardiac chambers.
Symptomatic or complicated aneurysms can be treated by surgery with excellent results, and the risk to asymptomatic patients is low, although associated complications (valvar aortic regurgitation, left ventricular dysfunction, aortic root and ascending aorta involvement, infection, and associated congenital cardiac malformations) may increase the risk.

Treatment of asymptomatic and uncomplicated sinus of Valsalva aneurysms is controversial. Opinion is divided between those who see surgery as a last resort and those who think it is necessary to prevent the development of potentially fatal complications. ${ }^{7-9}$ Currently, there are no follow up studies of asymptomatic and uncomplicated sinus of Valsalva aneurysms that could set a precedent for treatment of the condition. ${ }^{8}$

Furthermore, surgical management depends on whether the aneurysm has ruptured and whether there is aortic valvar insufficiency or ventricular septal defect. The aim of surgery is to close the aneurysmal sac, thus avoiding the creation of aortic valve dysfunction. ${ }^{10}$ If the aortic valve is involved and the coronary ostia originate in the aneurysm area, aortic valve and aortic root replacement may be necessary. ${ }^{12}$

Sinus of Valsalva aneurysms may be congenital or acquired through trauma, and although the latter are very rare, some cases have been described in which these aneuryms are seen in survivors of road traffic accidents. The aortic isthmus is often the most affected part of the body in accidents where injury has been caused as a direct result of deceleration: such lesions are rare in the aortic root, and often fatal. ${ }^{4}$

Thus, the discovery of this type of aneurysm in a healthy man with no history of heart disease, after an accident, favours a diagnosis of an aneurysm of acquired traumatic origin, and recently developed aortic insufficiency, despite the rare asymptomatic acute aortic regurgitation.

Nevertheless, the aneurysm and aortic insufficiency could have been congenital, with mild and asymptomatic regurgitation which had recently worsened and resulted in the heart murmur detected after the accident. The left ventricle was not dilated and, therefore, it is possible that this patient had been asymptomatic for a long time and that the aneurysm and aortic regurgitation were found coincidentally at the time of the crash. However, as no echocardiogram was taken before the road traffic accident, the pre-existence of the condition cannot be confirmed and the symptoms support the diagnosis of an aneurysm acquired through trauma.

We gratefully acknowledge the assistance of Mrs $M^{a}$ Júlia Ribeiro.

1 Simic O, Schneider B, Stein J, et al. Unruptured aneurysms of the non-coronary and left sinuses of Valsalva accompanied by severe aortic valve regurgitation. Eur $\mathcal{f}$ Cardiothoracic Surg 1996;10:1030-2.

2 Holman WL. Aneurysms of the sinuses of Valsalva. In: Sabiston DC, Spencer FC, eds. Surgery of the chest. Philadelphia: WB Saunders \& Co, 1995:1316-26. 
3 Friedman WF. Aortic sinus aneurysm and fistula. In: Braunwald $\mathrm{E}$, ed. Heart disease. A text book of cardiovas cular medicine. Philadelphia: WB Saunders \& Co, 1997

4 Gharzudine WS, Sawaya JI, Kazma HK, et al. Traumatic pseudoaneurysm of the left sinus of Valsalva: a case report. F Am Soc Echocardiography 1997;10:377-80.

5 Feigenbaum H. Sinus of Valsalva aneurysms. In: Feigenbaum H, ed. Echocardiography. Pennsylvania: Lea and Febiger, 1994:646-7.

6 Jain SP, Mahan EF, Nanda NC, et al. Doppler colour flow mapping in the diagnosis of sinus of Valsalva aneurysm. Echocardiography 1989;6:533-4.
7 Hiyamuta K, Ohtsuki T, Shimamatsu M, et al. Aneurysm of the left aortic sinus causing acute myocardial infarction. Circulation 1983;67:1151-4.

8 Jebara VA, Chavaud S, Portoghese M, et al. Isolated extracardiac unruptured sinus of Valsalva aneurysms. Ann Thorac Surg 1992;54:323-6.

9 Williams TG, Williams BT. Isolated unruptured aneurysm of the left coronary sinus of Valsalva. Ann Thorac Surg 1983;35:556-9.

10 Warnes CA, Maron B J, Jones M, et al. Asymptomatic sinus of valsalva aneurysm causing right ventricular outflow obstruction before and after rupture. Am f Cardiol 1984;84:1383-4. 